Received: 11 July 2018

Accepted: 18 October 2018

Published online: 31 October 2018

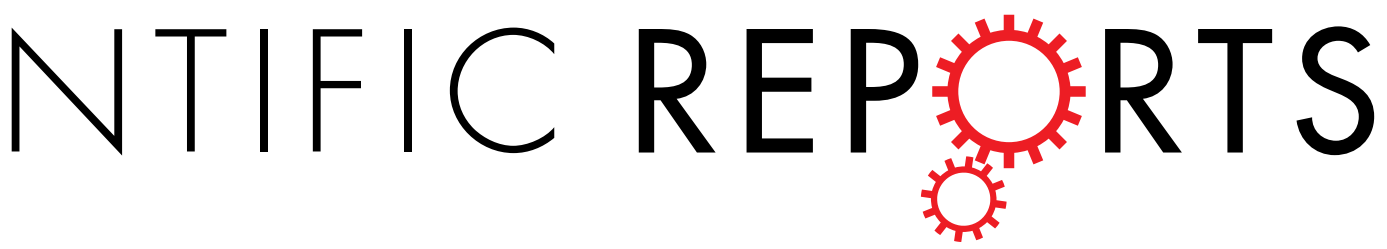

\title{
OPEN Thermotaxis in Chlamydomonas is brought about by membrane excitation and controlled by redox conditions
}

\author{
Masaya Sekiguchi, Shigetoshi Kameda, Satoshi Kurosawa, Megumi Yoshida \& \\ KenjiroYoshimura 1
}

Temperature is physiologically critical for all living organisms, which cope with temperature stress using metabolic and behavioral responses. In unicellular and some multicellular organisms, thermotaxis is a behavioral response to avoid stressful thermal environments and promote accumulation in an optimal thermal environment. In this study, we examined whether Chlamydomonas reinhardtii, a unicellular green alga, demonstrated thermotaxis. We found that between $10^{\circ} \mathrm{C}$ and $30^{\circ} \mathrm{C}$, Chlamydomonas cells migrated toward lower temperatures independent of cultivation temperature. Interestingly, when we applied reagents to change intracellular reduction-oxidation (redox) conditions, we saw that thermotaxis was enhanced, suppressed, or reversed, depending on the redox conditions and cultivation temperature. Thermotaxis was almost absent in ppr2 and ppr3 mutants, which cannot swim backward because of a defect in generating calcium current in flagella. The frequency of spontaneous backward swimming was lower at more favorable temperature, suggesting a pivotal role of spontaneous backward swimming generated by flagellar membrane excitation.

Temperature is a key environmental factor for living organisms because chemical reaction rates and physical characteristics of biological materials can change substantially with temperature. Living organisms acclimate to cold and heat stress using acquired mechanisms, including the ability to migrate to an environment with temperatures suitable for inhabitation. One of the simplest forms of the behavior to migrate to a suitable thermal environment is thermotaxis. Thermotaxis has been found in multicellular organisms, such as Caenorhabditis elegans and Drosophila melanogaster, as well as in unicellular organisms, such as Paramecium caudatum, Dictyostelium discoideum, Physarum polycephalum, and Escherichia coli ${ }^{1}$. Individual cells within multicellular organisms also show thermotaxis. For example, mammalian sperm migrate through the oviduct to the fertilization site guided by a rise in temperature ${ }^{2}$.

The investigation of how unicellular organisms migrate toward preferred temperatures began more than 100 years ago ${ }^{1}$. In particular, the thermotactic behavior of Paramecium cells has been well studied. Paramecium cells accumulate at sites that are close to the cultivation temperature, i. e. the temperature at which cells are grown ${ }^{1}$. Accumulation at these sites occurs because cells frequently reverse their swimming direction when they encounter a temperature change that deviates from the cultivation temperature and increase their swimming velocity when they experience a temperature change that approaches the cultivation temperature ${ }^{3,4}$. The reversal in swimming direction is induced by a depolarizing receptor potential, which triggers an action potential in the cilia ${ }^{5}$. These studies on Paramecium cells highlighted the thermotaxis in unicellular organisms more than 30 years ago, but the molecular mechanisms for thermoreception and signal transduction are not yet understood.

The understanding of the molecular mechanisms for thermotaxis has progressed greatly in recent years, from investigations of mammalian sperm. Human sperm migrates toward warmer temperatures, ranging from $29^{\circ} \mathrm{C}$ to $41^{\circ} \mathrm{C}^{2}$. Sperm can detect a temperature gradient as small as $0.014^{\circ} \mathrm{C} / \mathrm{mm}$, suggesting that sperm detect temporal changes in temperature rather than spatial differences ${ }^{2}$. Several molecules have been proposed to be sensor molecules, including opsin and transient receptor potential (TRP) channels such as TRPV1, TRPV4, and TRPM $8^{6-9}$.

Department of Machinery and Control Systems, College of Systems Engineering and Science, Shibaura Institute of Technology, Saitama, 337-8570, Japan. Correspondence and requests for materials should be addressed to K.Y. (email: kenjiroy@shibaura-it.ac.jp) 
TRP channels are multimodal sensor for thermal, chemical and mechanical stimuli, but the function of opsins as a thermosensor awaits to be established.

Temperature is a critical environmental factor also for Chlamydomonas cells, which produce small heat shock proteins, chaperonins, and HSP70 heat shock proteins, and also undergo other heat shock responses to cope with heat stress ${ }^{10-13}$. In response to a cold shock of $4^{\circ} \mathrm{C}$, cells halt proliferation and accumulate starch and sugar ${ }^{14}$. Behavioral responses to avoid stressful warm or cold environments are expected to be present in Chlamydomonas. Although C. moewusii cells are reported to migrate toward warmer temperatures in a $10^{\circ} \mathrm{C}-15^{\circ} \mathrm{C}$ gradient ${ }^{15}$, there has been no report in which the temperature range was systematically manipulated to examine a relationship with cultivation temperature.

In contrast to thermotaxis, phototaxis has been extensively studied in Chlamydomonas. Two flagella of Chlamydomonas beat in a breast-stroke like pattern during forward swimming and, during phototaxis, Chlamydomonas cells make by a turn toward or away from a light source by controlling the balance of the propulsive forces generated by the two flagella ${ }^{16,17}$. The balance depends on the intraflagellar calcium ion concentration; thus, loss of calcium-dependent control in ptx 1 mutants results in a phototaxis defect ${ }^{18-20}$. The direction of phototaxis in Chlamydomonas depends on the light intensity, but is also affected by intracellular reduction-oxidation (redox) conditions ${ }^{21}$. Cells migrate toward a light source when the light intensity is weak, but the direction reverses under reducing conditions. In contrast, cells swim away from light sources with strong intensity, but the direction reverses under oxidizing conditions. However, it is not fully understood how ubiquitous the influence of redox state is on cell behavior. Thus, in this study we investigate whether redox state affects thermotaxis.

Our use of $C$. reinhardtii for this study has the potential to advance the understanding of thermotaxis because a wide range of molecular biology tools, including the whole genome sequence, an indexed and mapped mutant library, and CRISPR/Cas9 gene editing method are available for this organism ${ }^{22-24}$. It is noteworthy that seven opsins and at least eight TRP channels are expressed in Chlamydomonas cells, considering the involvement in sperm thermotaxis ${ }^{25}$. Further, a large number of characterized Chlamydomonas mutants can support the analysis of the involvement of specific functions and genes in a given process.

In this study, we assessed thermotaxis in C. reinhardtii and examined how their movement correlated with the cultivation temperature. In contrast to Paramecium and C. moewusii, C. reinhardtii cells migrated toward lower temperatures between $10^{\circ} \mathrm{C}$ and $30^{\circ} \mathrm{C}$, irrespective of the cultivation temperature. We also tested whether the thermotaxis was affected by intracellular redox conditions and found that thermotaxis was greatly affected by redox states. Lastly, we used mutants defective for flagellar motility control to investigate the mechanism for thermotaxis.

\section{Results}

Negative thermotaxis occurs in various temperature gradients. Chlamydomonas cells are usually cultured at $25^{\circ} \mathrm{C}$ in laboratories, because they grow fastest around this temperature. When we generated a $5^{\circ} \mathrm{C}$ temperature gradient from $20^{\circ} \mathrm{C}$ to $25^{\circ} \mathrm{C}$ in a trough and placed the wild type cells in it, we found that cells accumulated toward the $20^{\circ} \mathrm{C}$ side (Fig. 1A). We evaluated the cell distribution by converting the cell density to gray scale and obtaining the mean gray scale for five sections. We observed an increase in cell density in two sections with lower temperatures $5 \mathrm{~min}$ after the onset of temperature gradient, whereas the cell density decreased in three sections with higher temperatures (Fig. 1B). We saw that this tendency progressed at least for $20 \mathrm{~min}$. The bias of cell distribution was expressed as thermotactic index, in which zero indicates that the distribution is even, and positive or negative values indicate that the distribution is biased toward higher or lower temperatures, respectively. The use of the thermotactic index confirmed that negative thermotaxis (i.e. migration toward lower temperatures) occurred during this period (Fig. 1C).

When cells cultured at $25^{\circ} \mathrm{C}$ were placed in a $5^{\circ} \mathrm{C}$ temperature gradient of $10^{\circ} \mathrm{C}-15^{\circ} \mathrm{C}, 15^{\circ} \mathrm{C}-20^{\circ} \mathrm{C}$, $20^{\circ} \mathrm{C}-25^{\circ} \mathrm{C}$, and $25^{\circ} \mathrm{C}-30^{\circ} \mathrm{C}$ gradients, cells demonstrated negative thermotaxis at all temperature levels (Fig. 2A). The magnitude of negative thermotaxis was comparable between $15^{\circ} \mathrm{C}-20^{\circ} \mathrm{C}, 20^{\circ} \mathrm{C}-25^{\circ} \mathrm{C}$, and $25^{\circ} \mathrm{C}-30^{\circ} \mathrm{C}$ gradients, but was smallest with the $10^{\circ} \mathrm{C}-15^{\circ} \mathrm{C}$ temperature gradient.

Because the direction of thermotaxis in Paramecium depends on the cultivation temperature ${ }^{1}$, we further examined thermotaxis in cells cultured at $15^{\circ} \mathrm{C}$ and $20^{\circ} \mathrm{C}$. Cells cultured at $15^{\circ} \mathrm{C}$ and $20^{\circ} \mathrm{C}$ also showed negative thermotaxis at all temperatures (Fig. 2B). However, the magnitude of movement was greater in cells grown at $15^{\circ} \mathrm{C}$ and modest in cells grown at $20^{\circ} \mathrm{C}$, compared to thermotaxis in cells grown at $25^{\circ} \mathrm{C}$. Thermotaxis in the $10^{\circ} \mathrm{C}-15^{\circ} \mathrm{C}$ temperature gradient was the weakest, for cells grown at any temperature.

To confirm that the bias in cell density was not caused by a physical artifact, such as convection, we applied the above protocol to an immotile mutant, $p f 17$. The cell density remained uniform for $20 \mathrm{~min}$, resulting in thermotactic index of practically zero (Fig. 2C).

Thermotaxis is affected by redox conditions. The direction of phototaxis of Chlamydomonas depends on light intensity, but is also affected by intracellular redox conditions ${ }^{21}$. When we subjected cells grown at $25^{\circ} \mathrm{C}$ to reducing conditions by addition of 1,3-dimethylthiourea (DMTU), thermotaxis was almost completely lost (Fig. 3A). Thermotaxis also diminished substantially when we subjected cells to oxidizing conditions by addition of tert-butyl hydroperoxide $(t-\mathrm{BOOH})$. This was not due to a loss of motility, because cells were motile, although with a reduced swimming velocity (see Fig. 4). When cells grown at $20^{\circ} \mathrm{C}$ were placed in reducing conditions, we observed positive thermotaxis in $15^{\circ} \mathrm{C}-20^{\circ} \mathrm{C}, 20^{\circ} \mathrm{C}-25^{\circ} \mathrm{C}$, and $25^{\circ} \mathrm{C}-30^{\circ} \mathrm{C}$ gradients (Fig. $3 \mathrm{~B}$ ). Conversely, negative thermotaxis was enhanced in oxidizing conditions. Thus, the direction of thermotaxis reversed, depending on the redox condition, as observed in phototaxis ${ }^{21}$. Additionally, cells grown at $15^{\circ} \mathrm{C}$ showed weaker negative thermotaxis in reducing and oxidizing conditions, compared with thermotaxis under ambient conditions (Fig. 3C).

Thus, the result shows a marked impact of redox balance and cultivation temperature on thermotaxis. 
A

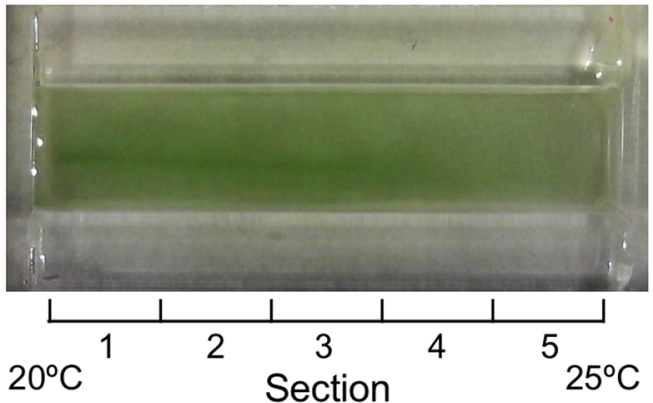

B

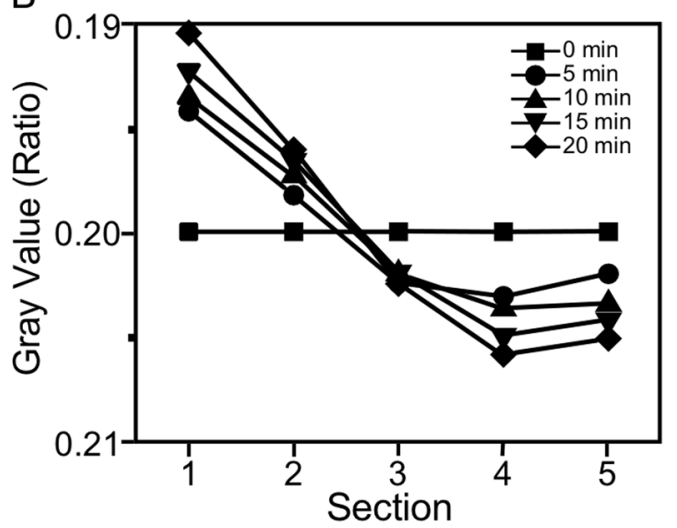

C

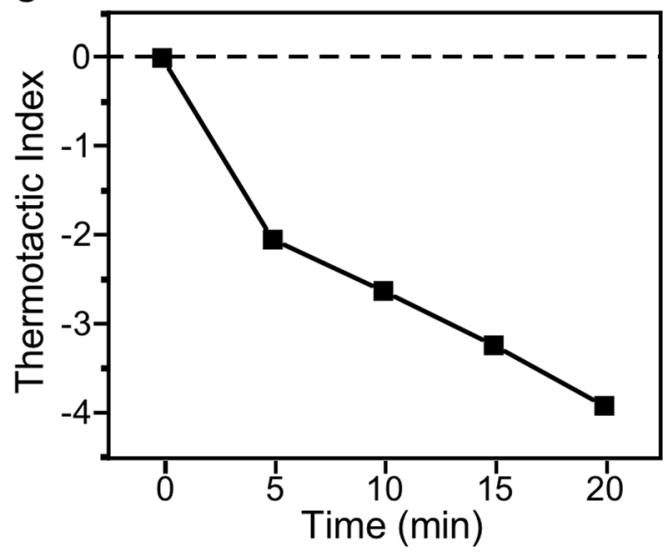

Figure 1. Response to $20^{\circ} \mathrm{C}-25^{\circ} \mathrm{C}$ temperature gradient in the wild type Chlamydomonas cells grown at $25^{\circ} \mathrm{C}$. (A) Top view of cell suspension in trough taken $20 \mathrm{~min}$ after cell suspension was placed under the temperature gradient. (B) Cell density along the length of the trough, divided into five sections. Cell density was obtained by gray scale and normalized to the average value at time $=0 \mathrm{~min}$. The density was obtained 0 to $20 \mathrm{~min}$ after incubation in the $20^{\circ} \mathrm{C}-25^{\circ} \mathrm{C}$ temperature gradient. Note that larger and smaller gray values (brighter and darker images) indicate less and more cells, respectively, and that the axis is inverted. (C) Time course of the changes in thermotactic index, using data shown in (B).

Swimming velocity. The observations above might result from low motility, which could cause cell accumulation ${ }^{26}$. To examine this possibility, we measured the swimming velocity of cells. We found that the swimming velocity of cells grown at $25^{\circ} \mathrm{C}$ increased with temperature from $42 \mu \mathrm{m} / \mathrm{s}$ to $123 \mu \mathrm{m} / \mathrm{s}$, in a range from $10^{\circ} \mathrm{C}$ to $30^{\circ} \mathrm{C}$ (Fig. 4A). In reducing conditions, the swimming velocity was slightly lower, and in oxidizing conditions, the velocity was less than half that of cells in ambient condition (e.g. 112, 82, and $45 \mu \mathrm{m} / \mathrm{s}$ in ambient, reducing, and oxidizing conditions, respectively, when assessed at $25^{\circ} \mathrm{C}$ ).

The effect of redox conditions on swimming velocity was almost negligible in cells grown at $20^{\circ} \mathrm{C}$ (Fig. $4 \mathrm{~B}$ ) (e.g. 83, 78, and $78 \mu \mathrm{m} / \mathrm{s}$ in ambient, reducing, and oxidizing conditions, respectively, when assessed at $25^{\circ} \mathrm{C}$ ). Therefore, the reversal or enhancement of thermotaxis in reducing or oxidizing conditions, respectively, is likely not attributable to a dependence of swimming velocity on temperature.

We found that cells grown at $15^{\circ} \mathrm{C}$ swam slightly faster in the reducing conditions than in oxidizing or ambient conditions (Fig. 4C) (e.g. 63, 94, and $79 \mu \mathrm{m} / \mathrm{s}$ in ambient, reducing, and oxidizing conditions, respectively, when assessed at $25^{\circ} \mathrm{C}$ ). Notably, cells in ambient condition showed pronounced thermotaxis in the $15^{\circ} \mathrm{C}-20^{\circ} \mathrm{C}$ gradient (Fig. 3C), but their swimming velocity did not differ between $15^{\circ} \mathrm{C}$ and $20^{\circ} \mathrm{C}$. Additionally, the temperature dependence of swimming velocity was very similar between the oxidizing and ambient conditions, despite a great difference in the magnitude of thermotaxis.

Theoretically, the density of moving particles is proportional to the inverse of speed ${ }^{26}$. The correlation of the inverse of swimming speed $(1 / v[\mathrm{~T}])$ with temperature $(\mathrm{T})$ supported overall tendency of higher cell density at lower temperatures (Supplementary Fig. 1 ). The ratio of $1 / v[\mathrm{~T}]$ with $5{ }^{\circ} \mathrm{C}$ difference, i. e. $(1 / v[\mathrm{~T}]) /$ $(1 / v[T+5])$, was calculated to estimate the accumulation toward lower temperatures (Supplementary Fig. 2). The ratio was almost exclusively larger than one, indicating accumulation toward lower temperature. However, the graph did not reproduce the remarkable thermotaxis in ambient condition in cells grown at $25^{\circ} \mathrm{C}$ and $15^{\circ} \mathrm{C}$ or positive thermotaxis in cells grown at $20^{\circ} \mathrm{C}$, confirming that thermotaxis cannot be explained fully by the temperature-dependent change in swimming velocity.

Thermotaxis is not observed in mutants with defects in flagellar motility control. Phototaxis in Chlamydomonas is mediated by calcium-dependent control of flagellar dominance, which steers the cell ${ }^{18}$. Here, we analyzed whether a similar flagellar dominance mechanism is involved in thermotaxis, by using a ptx 1 mutant 

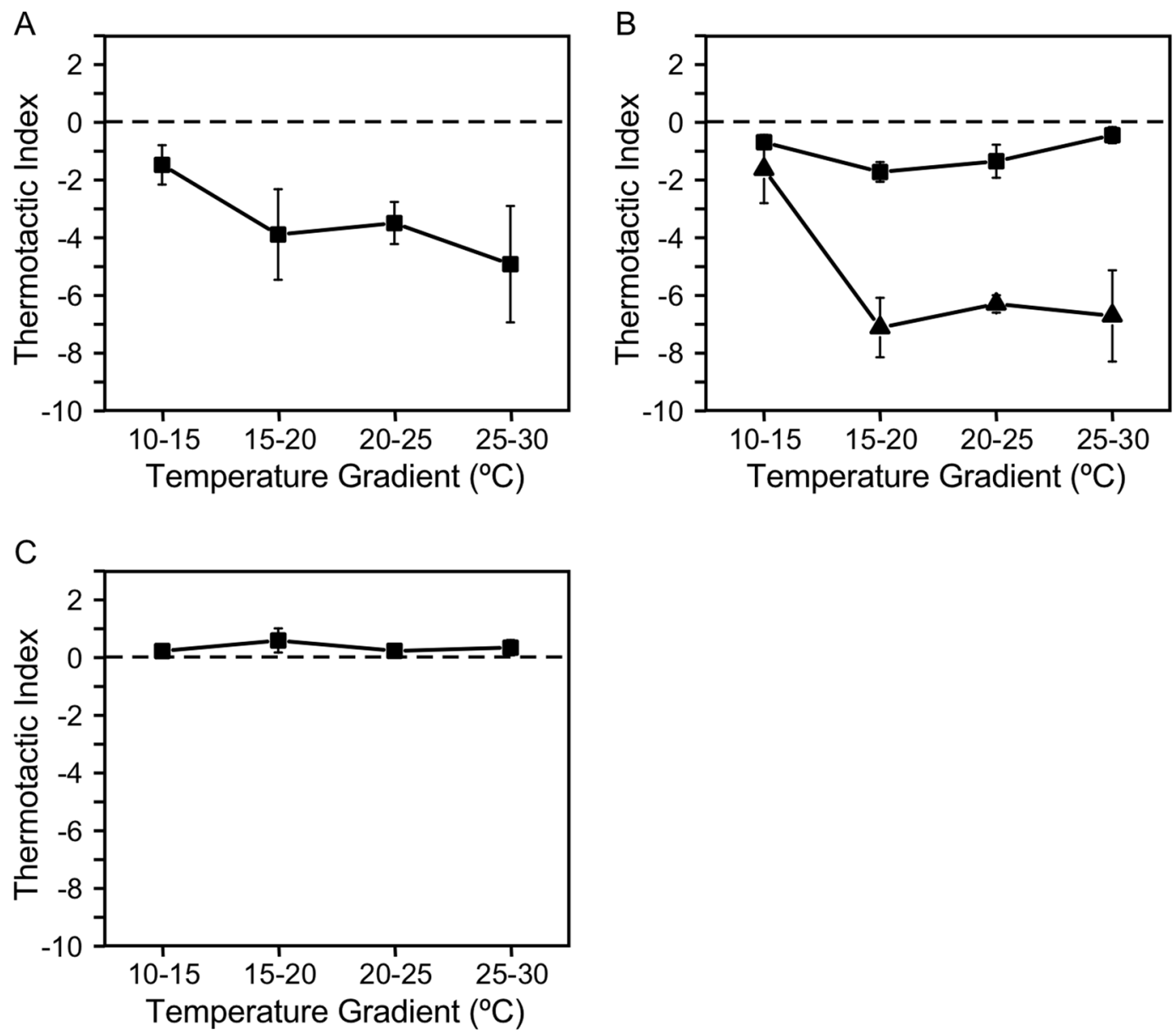

Figure 2. Responses to different temperature gradients in wild type cells grown at (A) $25^{\circ} \mathrm{C},(\mathbf{B}) 20^{\circ} \mathrm{C}$ (square), and $15^{\circ} \mathrm{C}$ (triangle). (C) Response of an immotile mutant, $p f 17$. Data represent mean \pm standard error of the mean (SEM) of three experiments.

that is defective for dominance contro $1^{19,20}$. When we placed ptx 1 cells grown at $25^{\circ} \mathrm{C}$ in a $5^{\circ} \mathrm{C}$ temperature gradient, we observed thermotaxis in the $20^{\circ} \mathrm{C}-25^{\circ} \mathrm{C}$ gradient (Fig. $5 \mathrm{~A}$ ). We also observed thermotaxis of ptx 1 cells in the $10^{\circ} \mathrm{C}-15^{\circ} \mathrm{C}, 15^{\circ} \mathrm{C}-20^{\circ} \mathrm{C}$, and $25^{\circ} \mathrm{C}-30^{\circ} \mathrm{C}$ gradients, although it was weaker than that of the wild type cells. Therefore, calcium-dependent control of flagellar dominance is probably not essential to thermotaxis.

In Paramecium, thermotaxis is mediated by the reversal of swimming direction when cells begin to swim away from their respective cultivation temperature. To examine the involvement of swimming reversal in Chlamydomonas thermotaxis, we used ppr2 and ppr3 mutants, which are defective for reversing swimming direc$\operatorname{tion}^{27,28}$. Chlamydomonas cells generate an all-or-none type flagellar current upon intense light or mechanical stimulation and convert flagellar bending pattern from the breast-stroke-like forward mode to the sinusoidal reverse mode ${ }^{29}$, but these mutants do not generate the flagellar current. When ppr 2 cells were grown at $25^{\circ} \mathrm{C}$ and placed in a $5^{\circ} \mathrm{C}$ temperature gradient, we did not observe thermotaxis in any gradient from $10^{\circ} \mathrm{C}$ to $30^{\circ} \mathrm{C}$ (Fig. 5B). We obtained a similar result with the ppr3 mutant.

The defect in thermotaxis in $p p r$ mutants suggests that backward swimming is involved in thermotaxis. Thus, we tested whether the frequency of spontaneous backward swimming changes with temperature. The distance between the slide and cover glass was kept at $\sim 100 \mu \mathrm{m}$ and the focus was set in the middle of them to exclude counting avoiding reaction, which occurs on collision with glass surface. We compared the cells grown at $25^{\circ} \mathrm{C}$ and kept in ambient redox condition and those grown at $20^{\circ} \mathrm{C}$ and kept in reducing condition since the former shows pronounced negative thermotaxis and the latter shows weak positive thermotaxis. The frequency of spontaneous backward swimming increased with temperature in the cells grown at $25^{\circ} \mathrm{C}$ and kept in ambient condition but, notably, the frequency did not increase with temperature in cells grown at $20^{\circ} \mathrm{C}$ and kept in reducing condition (Fig. 6A).

Since Paramecium cells increase the frequency of avoiding reaction when temperature changes away from the cultivation temperature, we observed spontaneous backward swimming during cooling and warming. If we assume that cells swim across a $5^{\circ} \mathrm{C}$ temperature gradient at speed of $100 \mu \mathrm{m} / \mathrm{s}$, they should experience a temporal temperature change rate of approximately $0.016^{\circ} \mathrm{C} / \mathrm{s}$. When the temperature was lowered from $30^{\circ} \mathrm{C}$ to $25^{\circ} \mathrm{C}$ at a rate of $0.01^{\circ} \mathrm{C} / \mathrm{s}$, the spontaneous backward swimming occurred at a rate as low as approximately $0.002 \mathrm{~s}^{-1}$ (Fig. 6B). When the temperature was increased from $25^{\circ} \mathrm{C}$ to $30^{\circ} \mathrm{C}$ at the same rate, spontaneous backward swimming occurred at a comparable frequency. These rates were lower than those at stationary temperature of $25^{\circ} \mathrm{C}$ 
A

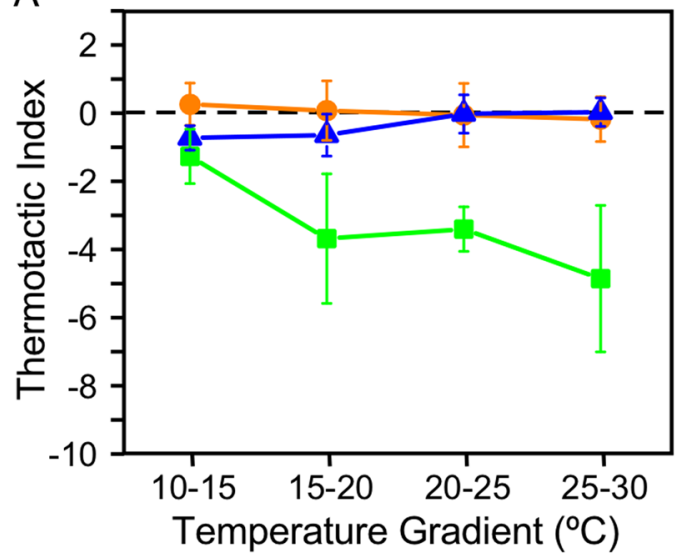

C

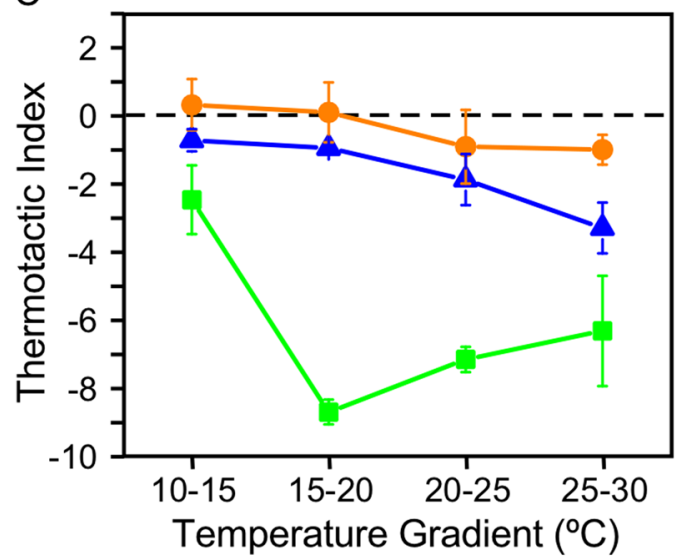

$\mathrm{B}$

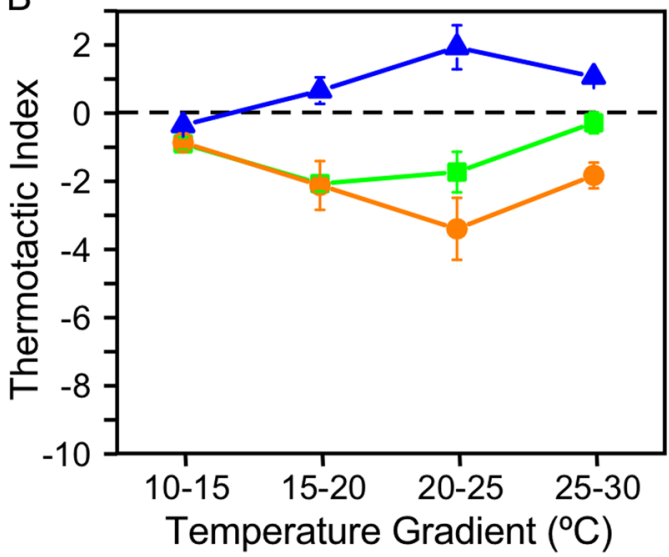

Figure 3. Responses to different temperature gradients with different redox conditions in wild type cells grown at $\left(\right.$ A) $25^{\circ} \mathrm{C},(\mathbf{B}) 20^{\circ} \mathrm{C}$, and $(\mathbf{C}) 15^{\circ} \mathrm{C}$. Thermotactic index of the data obtained under ambient (green), reducing (blue), and oxidizing (orange) conditions is shown. Data represent mean \pm SEM of three experiments.

and $30^{\circ} \mathrm{C}$ (Fig. 6A) suggesting that spontaneous backward swimming was suppressed when cells encountered a temporal temperature change. The suppression was more evident when the temperature was changed at a higher rate $\left(0.14^{\circ} \mathrm{C} / \mathrm{s}\right.$, Fig. $\left.6 \mathrm{C}\right)$.

The above observations imply that the frequency of spontaneous backward swimming changes with the temperature in accordance with the direction of thermotaxis at least in part, but does not depend on temporal temperature change. Since backward swimming is induced by a $\mathrm{Ca}^{2+}$ influx, it is possible that thermotaxis would not occur in a $\mathrm{Ca}^{2+}$-free medium. Such experiment was not presented here because the motility of Chlamydomonas cells was impaired in a $\mathrm{Ca}^{2+}$-free medium.

\section{Discussion}

Our experiments showed that Chlamydomonas cells migrated toward lower temperature in a range between $10^{\circ} \mathrm{C}$ and $30^{\circ} \mathrm{C}$. We also found that the redox state greatly affected thermotaxis. Notably, mutants defective in displaying backward swimming were deficient in thermotaxis.

It is unlikely that the biased cell distribution was due to a physical artifact, such as convection, based on following reasons. First, we observed that immotile mutants maintained a uniform distribution under the tested temperature gradients. Secondly, we observed different degrees and directions of bias under the same temperature gradient. For example, cells cultured at $15^{\circ} \mathrm{C}$ and $25^{\circ} \mathrm{C}$ showed a large distribution bias under ambient conditions, but showed minimal bias under reducing and oxidizing conditions. Cells cultured at $20^{\circ} \mathrm{C}$ migrated toward lower temperatures under oxidizing conditions, but toward higher temperatures under reducing conditions. These observations indicate that the bias is a physiological response rather than a physical artifact. It is also unlikely that the absence of thermotaxis in $p p r 2$ and $p p r 3$ was due to experimental error, because we observed clear thermotaxis in the positive control experiment on wild type cells, which used the same solution and trough on the same day.

Chlamydomonas cells favored lower temperatures in ambient redox conditions for all cultivation temperatures that were tested. Häder et al.$^{30}$ summarized that "Haematococcus and Volvox show negative thermotaxis moving to an optimum of $5^{\circ} \mathrm{C}$ to $10^{\circ} \mathrm{C}$," based on publications from 1893 and 1928. It is possible that the tendency to migrate to lower temperatures may be a common feature with the order Chlamydomonadales. This preference for lower temperature contrasts with thermotaxis in Paramecium, which accumulate at their respective cultivation 
A

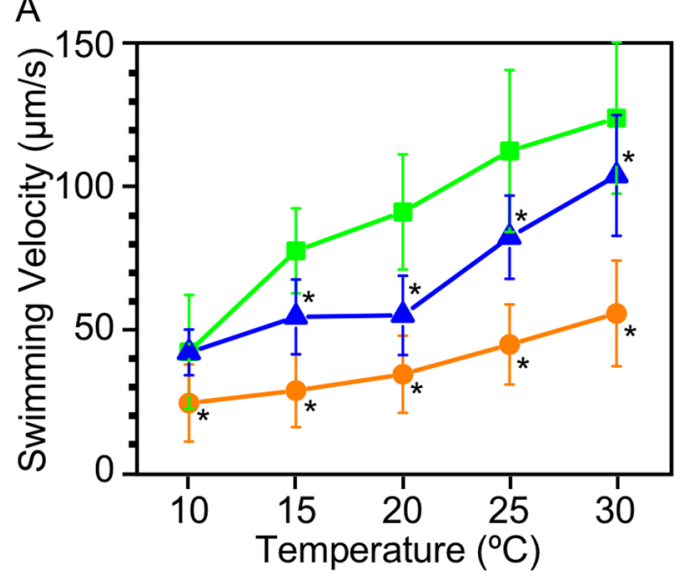

C

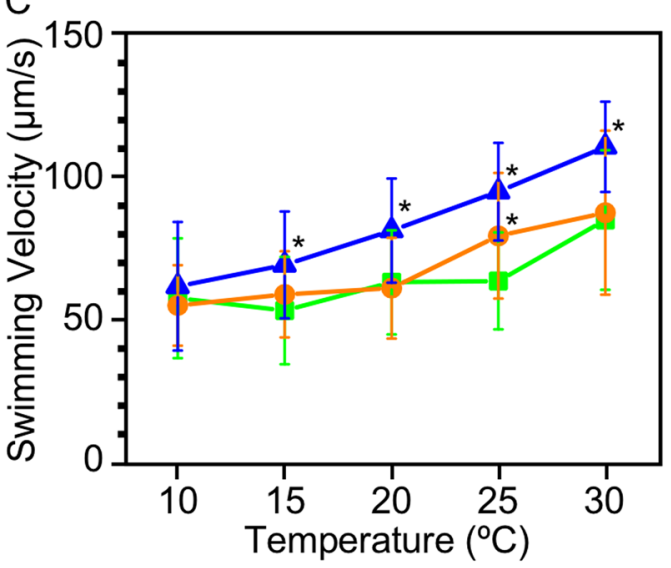

B

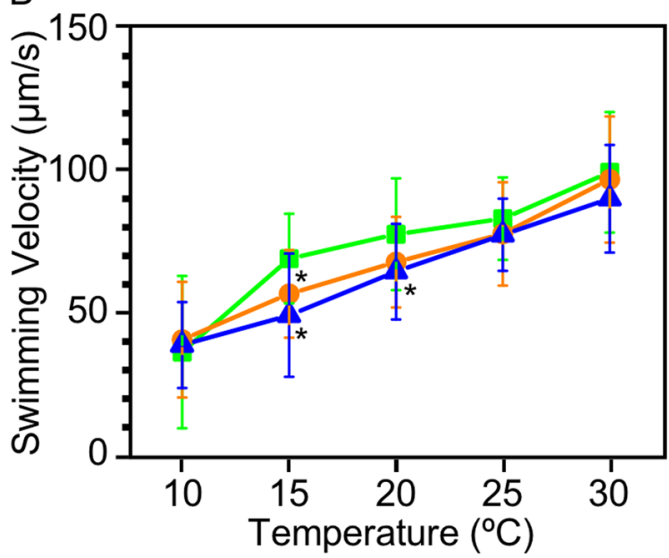

Figure 4. Swimming velocity at different temperatures in wild type cells grown at (A) $25^{\circ} \mathrm{C},(\mathbf{B}) 20^{\circ} \mathrm{C}$, and $(\mathbf{C})$ $15^{\circ} \mathrm{C}$. Data obtained under ambient (green), reducing, (blue), and oxidizing (orange) conditions are shown. Data represent mean $\pm S D$ of 30 cells. Asterisks indicate significant difference $(p<0.01, t$-test $)$ when compared with the data obtained in ambient condition.

A

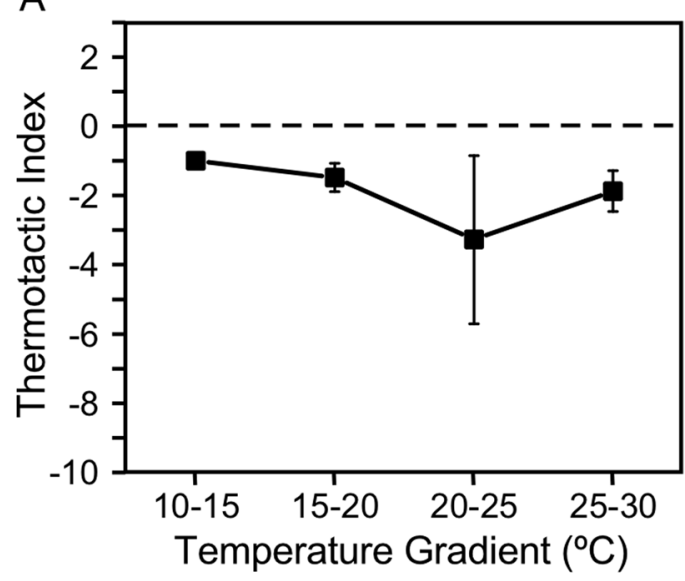

B

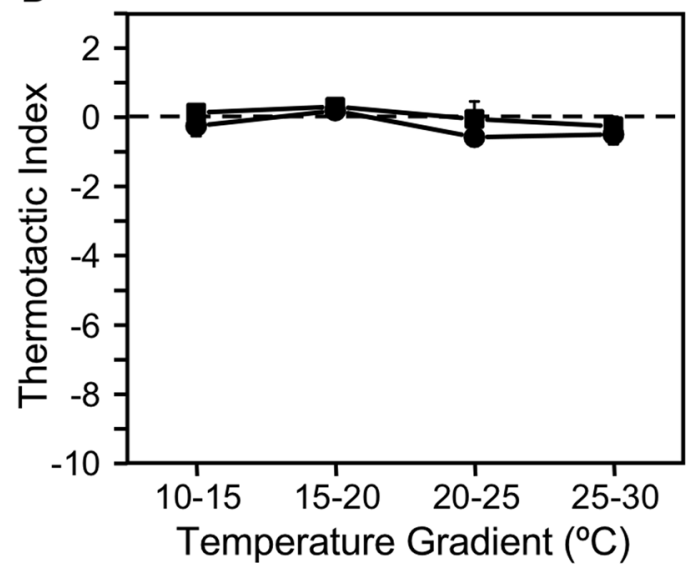

Figure 5. Response to temperature gradients in (A) a phototaxis mutant, $p t x 1$, and (B) photophobic-response mutants, ppr 2 (squares), and ppr3 (circle). Cells were grown at $25^{\circ} \mathrm{C}$. Data represent mean $\pm \mathrm{SEM}$ of three experiments. 
A

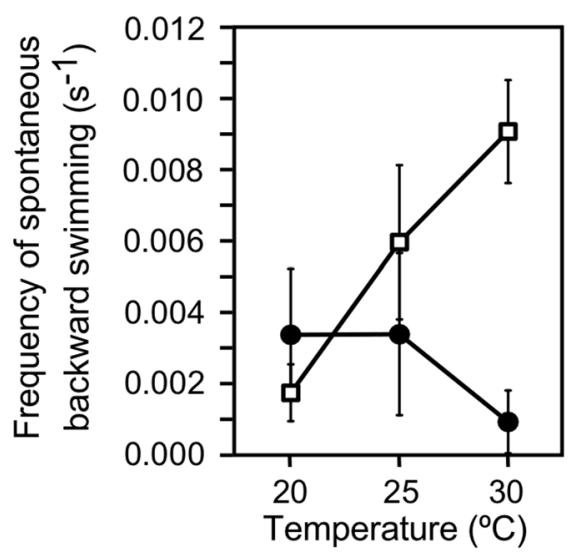

B

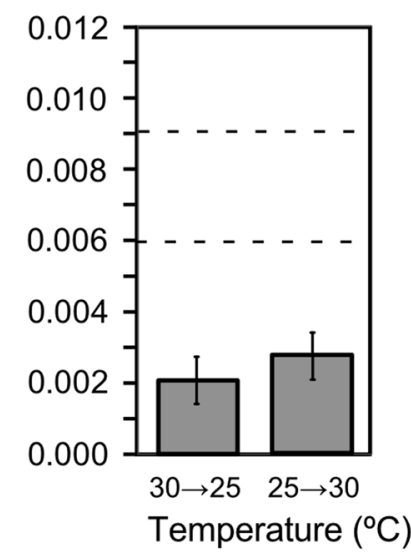

C

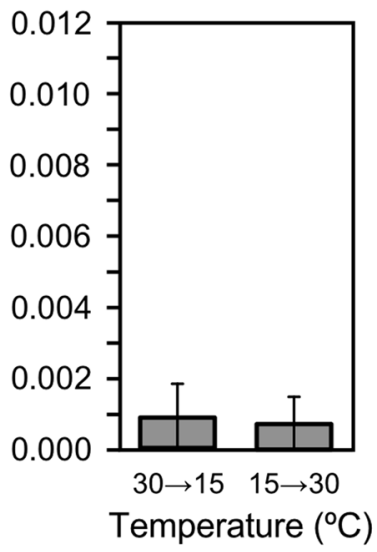

Figure 6. Frequency of spontaneous backward swimming. (A) Temperature was kept constant. Cells were grown at $25^{\circ} \mathrm{C}$ and examined in ambient condition (open squares) and grown at $20^{\circ} \mathrm{C}$ and examined in reducing condition (closed circles). (B) Temperature was changed temporally from $30^{\circ} \mathrm{C}$ to $25^{\circ} \mathrm{C}$ and vice versa at a rate of $\pm 0.01{ }^{\circ} \mathrm{C} / \mathrm{s}$. Cells were grown at $25^{\circ} \mathrm{C}$. The upper and lower dashed lines show the frequency obtained at constant temperatures of $30^{\circ} \mathrm{C}$ and $25^{\circ} \mathrm{C}$ as shown in A. (C) Temperature was changed from $30^{\circ} \mathrm{C}$ to $15^{\circ} \mathrm{C}$ and vice versa at a rate of $\pm 0.14^{\circ} \mathrm{C} / \mathrm{s}$. Data represent mean $\pm \mathrm{SEM}$ of ten experiments in $\mathrm{A}$ and six experiments in $\mathrm{B}$ and $\mathrm{C}$.

exposure to temperatures ranging from $4{ }^{\circ} \mathrm{C}$ to $9^{\circ} \mathrm{C}$, Chlamydomonas cell division pauses and cells accumulate starch and sugar for acclimation ${ }^{14,32}$. Thus, Chlamydomonas cells likely migrate toward low temperatures, as long as they can continue to proliferate but do not prefer the low temperature at which cell division halts.

Redox balance is altered by external factors and cellular activities, such as respiration and photosynthesis, and is detected by various signaling proteins, including ion channels and kinases ${ }^{33}$. The perturbation of redox balance could thus affect cell behavior. For example, Chlamydomonas cells display negative phototaxis under reducing conditions and positive phototaxis under oxidizing conditions ${ }^{21}$. Redox state also controls bacterial chemotaxis by alteration of CheA kinase activity ${ }^{34}$. In this study, we found that shifting the redox balance to reducing and oxidizing extremes suppressed thermotaxis in Chlamydomonas, except in cells grown at $20^{\circ} \mathrm{C}$. When the phototaxis result is compared with thermotaxis data obtained at comparable temperature $\left(25^{\circ} \mathrm{C}\right)$, the decline in thermotaxis in reducing and oxidizing conditions contrasts with augmentation of phototaxis at these extremes. Chlamydomonas cells appear to shut off thermotaxis and give priority to phototaxis in unusually reduced or oxidized states.

The possible causes for the observed biased distribution along the experimental temperature gradient include: (1) low motility at the site of cell accumulation; (2) change in the frequency of spontaneous backward swimming and/or swimming velocity; (3) steering of the swimming direction toward the preferred temperature. The second mechanism is involved in Paramecium and bacterial thermotaxis. The third mechanism is responsible for phototaxis in Chlamydomonas. We discuss these possibilities below.

On the whole, slower swimming at lower temperature is likely to contribute to negative thermotaxis because cells stay for a longer period where temperature is $\operatorname{low}^{26}$. Consistent with this idea, C. moewusii cells, which show positive thermotaxis, swim slowest at highest temperature examined ${ }^{15}$. However, thermotaxis is not fully attributable to swimming velocity. This is because the direction of thermotaxis reversed depending on redox state in cells grown at $20^{\circ} \mathrm{C}$ without changing the sign of the slope in the relationship between temperature and swimming velocity. Additionally, cells grown at $15^{\circ} \mathrm{C}$ exhibited a stronger thermotaxis response under ambient conditions than under oxidizing or reducing conditions, although the temperature dependency of swimming velocity did not significantly change. Lastly, the thermotactic profile predicted from swimming velocity did not reproduce the actual thermotactic profile.

ptx 1 cells showed thermotaxis, indicating that cell steering caused by $\mathrm{Ca}^{2+}$-dependent control of flagellar dominance is not involved in thermotaxis. The finding that thermotaxis does not require reorientation mechanism indicates that this behavior does not fall into "taxis" in a narrow sense, in which cells turn toward or away from stimulus as in phototaxis in Chlamydomonas ${ }^{17}$.

On the other hand, the absence of thermotaxis in ppr2 and ppr3 indicates that backward swimming or components used in backward swimming is involved in thermotaxis. The reversal of swimming direction in Chlamydomonas cells is induced by a $\mathrm{Ca}^{2+}$ influx through a voltage dependent $\mathrm{Ca}^{2+}$ channel in the flagellar membrane, but this flagellar current is absent in $p p r 2$ and $p p r 3$ mutants ${ }^{27,28}$. Interestingly, the frequency of spontaneous backward swimming increased with temperature in a condition where negative thermotaxis occurs but not under weak positive thermotaxis condition. Since pivoting motion at the end of backward swimming randomizes the direction of swimming, it is plausible that cells far from preferred temperature gain a higher chance to alter the swimming direction than the cells close to preferred temperature. The spontaneous backward swimming was, however, suppressed in Chlamydomonas cells when temperature was changed temporally. Thus, Chlamydomonas cells probably respond to static temperature rather than a temporal change in temperature whereas Paramecium 
and bacterial cells respond to a temporal change in temperature ${ }^{3,4,35,36}$. Suppression of spontaneous backward swimming upon temporal change in temperature suggests that swimming path would not be interrupted by spontaneous backward swimming when cells swim parallel to the temperature gradient but that they undergo spontaneous backward swimming more frequently when they swim vertically to the gradient.

It is of interest how Chlamydomonas cells detect temperature. The $0.14^{\circ} \mathrm{C} / \mathrm{mm}$ gradient used in this study is not subtle, considering that sperm undergo thermotaxis with a $0.014^{\circ} \mathrm{C} / \mathrm{mm}$ temperature gradient. Sperm thermotaxis involves TRP channels and opsins $s^{6-9}$. Chlamydomonas cells also express multiple TRP channel and opsin genes. At least eight TRP channels are expressed, including PKD2, TRP11, and ADF1, which are involved in mating, mechanoreception, and deflagellation, respectively ${ }^{25,37,38}$. Eight opsins are expressed, including the channelopsins, ChR1 and ChR2, which generate photoreceptor current at the eyespot ${ }^{39,40}$. However, the activity of TRP channels, opsins, and other proteins in thermosensing requires further study.

This study has shown that Chlamydomonas cells migrate predominantly toward the lower temperature in ambient condition. Thermotaxis is not a simple response to temperature, but is facilitated, inhibited, or even inverted by physiological conditions such as redox state and cultivation temperature. Our work sheds light on the dominance of redox balance on how cells control their motility and decide behavioral strategy.

\section{Methods}

Cell and culture condition. We used Chlamydomonas reinhardtii $137 \mathrm{c} \mathrm{mt}+(\mathrm{CC} 125)$ as wild type in our study. We also used the following mutant strains. $p f 17$ is a mutant with paralyzed flagella ${ }^{41}$. ppr 2 and $p p r 3$ are mutants that do not show photophobic responses ${ }^{27}$. ptx 1 is a mutant defective in phototaxis ${ }^{19}$. Cells were grown in Sager and Granick liquid culture medium ${ }^{42}$ under 12 -hour light, 12 -hour dark cycle at $15^{\circ} \mathrm{C}, 20^{\circ} \mathrm{C}$, or $25^{\circ} \mathrm{C}$ for 3 or 4 days.

We washed cells twice with a solution containing: $1 \mathrm{mM} \mathrm{KCl}, 0.3 \mathrm{mM} \mathrm{CaCl}_{2}, 0.2 \mathrm{mM}$ EGTA-KOH, and $5 \mathrm{mM}$ HEPES-KOH ( $\mathrm{pH} 7.4$ ). The cell density was adjusted to $3 \times 10^{6} \mathrm{cells} / \mathrm{ml}$. Cells were kept at the cultivation temperature throughout the preparation process.

We included $0.2 \mathrm{mM} t$-BOOH or $75 \mathrm{mM}$ DMTU in the experimental solution to assess thermotaxis under oxidizing or reducing conditions, respectively, according to Wakabayashi et al. ${ }^{21}$.

Thermotaxis assay. We generated the temperature gradient on a $0.3-\mathrm{mm}$-thick brass plate that was placed between two Peltier devices (Supplementary Fig. 3). We monitored the temperature on the Peltier devices using platinum temperature sensors and controlled the temperature with a digital proportional integral controller (PLC-24V6A, T. S. Laboratory, Japan). We placed two complementary metal oxide semiconductor (CMOS) temperature sensors at positions that trisect the trough to check the temperature gradient along the brass plate. As shown in Supplementary Table 1, the temperatures at the CMOS sensors confirmed that a continuous temperature gradient was generated between Peltier devices. A water-soaked filter paper was placed between the brass plate and trough for better thermal conductivity, as well as providing a uniform matte background for observation. The bottom of the trough was made of a thin glass $(0.12$ to $0.17 \mathrm{~mm}$ thick $)$ and the inside dimensions of the trough were $31 \times 5 \times 5 \mathrm{~mm}$ (length $\times$ width $\times$ height). The brass plate and trough were covered with Styrofoam for thermal insulation. The entire experimental set-up was placed in a dark box.

For the thermotaxis assay, we placed $1.1 \mathrm{ml}$ of cell suspension in the trough at cell density of $3 \times 10^{6} \mathrm{cells} / \mathrm{mL}$. We illuminated the cells with red light $(\lambda>600 \mathrm{~nm})$ because cell motility tends to decline in total darkness. Red light does not promote phototaxis ${ }^{43}$. We changed the direction of the temperature gradient between experiments to avoid the influence of factors independent of the temperature gradient.

We recorded the cell distribution with a charge-coupled device (CCD) camera and converted the image to gray scale (larger and smaller value indicate brighter and darker images, i. e. less and more cells, respectively). Gray scale value changed almost linearly with cell concentration in a range from $1.3 \times 10^{5}$ to $1.2 \times 10^{7}$ cells $/ \mathrm{mL}$ (Supplementary Fig. 4). We divided the trough into five sections along its length and averaged the gray scale of each section. The value was normalized to 1 with the average gray scale at time $=0 \mathrm{~min}$, to yield $\mathrm{G}_{\mathrm{i}}(\mathrm{i}=1$ to 5$)$. The percent cell density in each section was expressed as

$$
C i=100 \times\left\{1+\left(1-\frac{5 G i}{\sum_{j=1}^{5} G j}\right)\right\}
$$

Thermotactic index (TI) was defined as

$$
T I=\frac{\sum_{i=1}^{5} S i C i}{5}
$$

where $S_{i}$ is 2 (highest temperature), $1,0,-1$, and -2 (lowest temperature) in the order of sections with higher temperature. Thus, positive TI represents accumulation toward warmer sections (positive thermotaxis).

Swimming velocity. We recorded swimming velocity at different temperatures using an inverted microscope (IX70, Olympus) equipped with a temperature-controlled stage (Thermo Plate, Tokai Hit, Japan). We acquired the video using a CCD camera (XC-EI50, Sony) connected to a personal computer (Apple Computer) through a video converter (ADVC-55, Canopus, Japan). We used red light $(\lambda>600 \mathrm{~nm})$ for illumination to avoid a photoresponse $\mathrm{e}^{43}$. Swimming velocity was calculated by dividing displacement of a cell by the time of measurement using ImageJ. 
The frequency of spontaneous backward swimming was recorded in the same way except that the distance between the slide and cover glass was set at approximately $100 \mu \mathrm{m}$ by adjusting the volume of cell suspension. We set the focus in the middle between the slide and cover glass so as not to count the avoiding reaction, which occurs when cells collided with the glass surface.

\section{Data Availability}

All data generated or analyzed during this study are included in this published article and the Supplementary Information.

\section{References}

1. Jenning, H. S. Behavior of the lower organisms. Columbia University Biological Series X. Columbia University Press (1906).

2. Bahat, A. et al. Thermotaxis of mammalian sperm cells: a potential navigation mechanism in the female genital tract. Nat. Med. $\mathbf{9}$, 149-150 (2003)

3. Tawada, K. \& Oosawa, F. Responses of Paramecium to temperature change. J. Protozool. 19, 53-57 (1972)

4. Nakaoka, Y. \& Oosawa, F. Temperature-sensitive behavior of Paramecium caudatum. J. Protozool. 24, 575-580 (1977).

5. Hennessey, T. M., Saimi, Y. \& Kung, C. A heat-induced depolarization of Paramecium and its relationship to thermal avoidance behavior. J. Comp. Physiol. 153, 39-46 (1983).

6. Pérez-Cerezales, S. et al. Involvement of opsins in mammalian sperm thermotaxis. Sci. Rep. 5, 16146 (2015).

7. De Toni, L. et al. Heat sensing receptor TRPV1 is a mediator of thermotaxis in human spermatozoa. PLoS One 11, e0167622 (2016).

8. Hamano, K., Kawanishi, T., Mizuno, A., Suzuki, M. \& Takagi, Y. Involvement of Transient Receptor Potential Vanilloid (TRPV) 4 in mouse sperm thermotaxis. J. Reprod Dev. 62, 415-422 (2016)

9. De Blas et al. TRPM8, a versatile channel in human sperm. PLoS One 4, e6095 (2009).

10. Kloppstech, K., Meyer, G., Schuster, G. \& Ohad, I. Synthesis, transport and localization of a nuclear coded 22-kd heat-shock protein in the chloroplast membranes of peas and Chlamydomonas reinhardi. EMBO J. 4, 1901-1909 (1985).

11. van Lis, R., Atteia, A., Mendoza-Hernández, G. \& González-Halphen, D. Identification of novel mitochondrial protein components of Chlamydomonas reinhardtii. A proteomic approach. Plant Physiol. 132, 318-330 (2003)

12. von Gromoff, E. D., Treier, U. \& Beck, C. F. Three light-inducible heat shock genes of Chlamydomonas reinhardtii. Mol. Cell Biol. 9, 3911-3918 (1989).

13. Schroda, M., Hemme, D. \& Mühlhaus, T. The Chlamydomonas heat stress response. Plant J. 82, 466-480 (2015).

14. Valledor, L., Furuhashi, T., Hanak, A. M. \& Weckwerth, W. Systemic cold stress adaptation of Chlamydomonas reinhardtii. Mol. Cell Proteomics. 12, 2032-2047 (2013).

15. Clegg, M. R., Maberly, S. C. \& Jones, R. I. Behavioural responses of freshwater phytoplanktonic flagellates to a temperature gradient. Eur. J. Phycol. 38, 195-203 (2003).

16. Foster, K. W. \& Smyth, R. D. Light Antennas in phototactic algae. Microbiol. Rev. 44, 572-630 (1980).

17. Isogai, N., Kamiya, R. \& Yoshimura, K. Dominance between the two flagella during phototactic turning in. Chlamydomonas. Zool. Sci. 17, 1261-1266 (2000)

18. Kamiya, R. \& Witman, G. B. Submicromolar levels of calcium control the balance of beating between the two flagella in demembranated models of Chlamydomonas. J. Cell Biol. 98, 97-107 (1984).

19. Horst, C. J. \& Witman, G. B. ptx1, a nonphototactic mutant of Chlamydomonas, lacks control of flagellar dominance. J. Cell Biol. 120, 733-741 (1993).

20. Okita, N., Isogai, N., Hirono, M., Kamiya, R. \& Yoshimura, K. Phototactic activity in Chlamydomonas 'non-phototactic' mutants deficient in $\mathrm{Ca}^{2+}$-dependent control of flagellar dominance or in inner arm dynein. J. Cell Sci. 118, 529-537 (2005).

21. Wakabayashi, K., Misawa, Y., Mochiji, S. \& Kamiya, R. Reduction-oxidation poise regulates the sign of phototaxis in Chlamydomonas reinhardtii. Proc. Natl. Acad. Sci. USA 108, 11280-11284 (2011).

22. Marchant, S. S. et al. The Chlamydomonas genome reveals the evolution of key animal and plant functions. Science 318, 245-250 (2007).

23. Li, X. et al. An indexed, mapped mutant library enables reverse genetics studies of biological processes in Chlamydomonas reinhardtii. Plant Cell. 28, 367-387 (2016).

24. Greiner, A. et al. Targeting of photoreceptor genes in Chlamydomonas reinhardtii via Zinc-Finger Nucleases and CRISPR/Cas9. Plant Cell 29, 2498-2518 (2017)

25. Fujiu, K., Nakayama, Y., Iida, H., Sokabe, M. \& Yoshimura, K. Mechanoreception in motile flagella of Chlamydomonas. Nat. Cell. Biol. 13, 630-632 (2011).

26. Schnitzer, M. J. Theory of continuum random walks and application to chemotaxis. Phys Rev E. 48, 2553-2568 (1993).

27. Matsuda, A., Yoshimura, K., Sineshchekov, O., Hirono, M. \& Kamiya, R. Isolation and characterization of novel Chlamydomonas mutants that display phototaxis but not photophobic response. Cell Motil. Cytoskel. 41, 353-362 (1998).

28. Fujiu, K., Nakayama, Y., Yanagisawa, A., Sokabe, M. \& Yoshimura, K. Chlamydomonas CAV2 encodes a voltage-dependent calcium channel required for the flagellar waveform conversion. Curr. Biol. 19, 133-139 (2009).

29. Harz, H., Nonnengässer, C. \& Hegemann, P. The photoreceptor current of the green alga. Chlamydomonas. Phil. Trans. R. Soc. Lond. B 338, 38-52 (1992).

30. Häder, D. -P., Hemmerbach, R. \& Lebert, M. Gravity and the behavior of unicellular organisms. Cambridge University Press (2005).

31. Maikova, A., Zalutskaya, Z., Lapina, T. \& Ermilova, E. The HSP70 chaperone machines of Chlamydomonas are induced by cold stress. J. Plant Physiol. 204, 85-91 (2016)

32. Hema, R., Senthil-Kumar, M., Shivakumar, S., Chandrasekhara Reddy, P. \& Udayakumar, M. Chlamydomonas reinhardtii, a model system for functional validation of abiotic stress responsive genes. Planta 226, 655-670 (2007).

33. D'Autreaux, B. \& Toledano, M. B. ROS as signaling molecules: mechanisms that generate specificity in ROS homeostasis. Nat. Rev. Mol. Cell Biol. 8, 813-824 (2007).

34. Samanta, D., Widom, J., Borbat, P. P., Freed, J. H. \& Crane, B. R. Bacterial energy sensor Aer modulates the activity of the chemotaxis kinase CheA based on the redox state of the flavin cofactor. J. Biol. Chem. 291, 25809-25814 (2016).

35. Adler, J. The sensing of chemicals by bacteria. Sci. Am. 234, 40-47 (1976).

36. Maeda, K., Imae, Y., Shioi, J.-I. \& Oosawa, F. Effect of temperature on motility and chemotaxis of Escherichia coli. J. Bacteriol. 127, 1039-1046 (1976).

37. Huang, K. et al. Function and dynamics of PKD2 in Chlamydomonas reinhardtii flagella. J. Cell Biol. 179, 501-514 (2007).

38. Hilton, L. K. et al. A forward genetic screen and whole genome sequencing identify deflagellation defective mutants in Chlamydomonas, including assignment of ADF1 as a TRP channel. G3 (Bethesda) 6, 3409-3418 (2016).

39. Nagel, G. et al. Channelrhodopsin-1: A light-gated proton channel in green algae. Science 296, 2395-2398 (2002).

40. Sineshchekov, O. A., Jung, K. H. \& Spudich, J. L. Two rhodopsins mediate phototaxis to low- and high-intensity light in Chlamydomonas reinhardtii. Proc. Natl. Acad. Sci. USA 99, 8689-8694 (2002).

41. Huang, B., Piperno, G., Ramanis, Z. \& Luck, D. J. Radial spokes of Chlamydomonas flagella: genetic analysis of assembly and function. J. Cell Biol. 88, 80-88 (1981) 
42. Sager, R. \& Granick, S. Nutritional studies with Chlamydomonas reinhardi. Ann. N. Y. Acad. Sci. 56, 831-838 (1953). 43. Moss, A. G., Pazour, G. J. \& Witman, G. B. Assay of Chlamydomonas phototaxis. Methods Cell Biol. 47, 281-287 (1995).

\section{Acknowledgements}

This study was supported by Japan Agency for Medical Research and Development (AMED/PRIME) under Grant Number JP18gm5810013h0004.

\section{Author Contributions}

M.S. and S.Ku. made the experimental setup for thermotaxis. M.S., S.Ka., S.Ku. performed themotaxis experiments. M.S. measured swimming velocity. S.Ka. collected backward swimming data. M.Y. and K.Y. gave technical advice. K.Y. designed and supervised the study. K.Y. wrote the main manuscript and all authors reviewed the manuscript. M.S., S.K., and K.Y. prepared figures.

\section{Additional Information}

Supplementary information accompanies this paper at https://doi.org/10.1038/s41598-018-34487-4.

Competing Interests: The authors declare no competing interests.

Publisher's note: Springer Nature remains neutral with regard to jurisdictional claims in published maps and institutional affiliations.

(c) (i) Open Access This article is licensed under a Creative Commons Attribution 4.0 International

License, which permits use, sharing, adaptation, distribution and reproduction in any medium or format, as long as you give appropriate credit to the original author(s) and the source, provide a link to the Creative Commons license, and indicate if changes were made. The images or other third party material in this article are included in the article's Creative Commons license, unless indicated otherwise in a credit line to the material. If material is not included in the article's Creative Commons license and your intended use is not permitted by statutory regulation or exceeds the permitted use, you will need to obtain permission directly from the copyright holder. To view a copy of this license, visit http://creativecommons.org/licenses/by/4.0/.

(C) The Author(s) 2018 